\title{
Personalidade e cognição: um estudo em idosas brasileiras
}

\author{
Personalidad y cognición: un estudio en mujeres brasileñas de edad avanzada
}

\author{
Personality and cognition: a study in Brazilian elderly women
}

Universidade Católica de Brasília (UCB), Brasília - DF/Brasil

ORCID: 0000-0002-1080-6353

E-mail: caio.psic23@gmail.com

\author{
Isabelle Patriciá Freitas Soares Chariglione \\ Universidade de Brasília (UnB), Brasília - DF/Brasil \\ ORCID: 0000-0001-8627-3736 \\ E-mail: ichariglione@gmail.com
}

\begin{abstract}
Resumo
O objetivo foi analisar os efeitos de diferentes intervenções cognitivas em dois grupos de idosas, além de identificar os

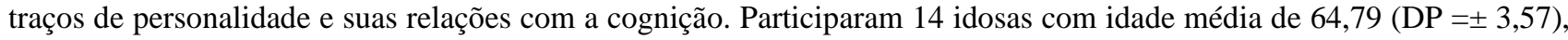
com ensino médio completo ou superior. Essas idosas eram pertencentes a um grupo de intervenções cognitivas e físicas e foram avaliadas em dois momentos (pré-intervenção e pós-intervenção), individualmente por meio de uma anamnese, medidas cognitivas (exame cognitivo de Addenbrooke - versão revisada) e de personalidade (Bateria Fatorial de Personalidade). Após a primeira avaliação, as participantes foram divididas em grupos de estimulação e treino cognitivo, e posteriormente, para a análise dos resultados, optou-se por reagrupar as participantes em duas categorias, sendo as que mantiveram/melhoraram ou pioraram nos resultados. Os resultados indicaram que escores baixos dos traços de personalidade de instabilidade, extroversão e interações sociais apresentaram ser significativos com as participantes que mantiveram ou melhoraram no momento pós-intervenção. Identificou-se a possibilidade de se incluir as diferenças individuais na construção e condução de práticas que envolvam intervenções cognitivas, visando uma maior eficácia das técnicas utilizadas na área da saúde.
\end{abstract}

Palavras-chaves: Avaliação psicológica; Envelhecimento; Saúde.

\section{Resumen}

El objetivo fue analizar los efectos de diferentes intervenciones cognitivas en dos grupos de mujeres de edad avanzada, además de identificar los rasgos de personalidad y su relación con la cognición. Participaron 14 mujeres de edad avanzada con una edad media de $64.79(\mathrm{DE}= \pm 3.57)$, con educación secundaria completa o superior. Estas mujeres mayores pertenecían a un grupo de intervenciones cognitivas y físicas y fueron evaluadas en dos momentos (preintervención y posintervención), individualmente a través de una anamnesis, medidas cognitivas (examen cognitivo de Addenbrooke - versión revisada) y personalidad (Batería Factorial de la Personalidad). Después de la primera evaluación, los participantes se dividieron en grupos de estimulación y entrenamiento cognitivo, y luego, para el análisis de los resultados, se decidió reagrupar a los participantes en dos categorías, las que mantenían / mejoraban o empeoraban los resultados. Los resultados indicaron que los puntajes bajos de los rasgos de personalidad de inestabilidad, extraversión e interacciones sociales fueron significativos con los participantes que mantuvieron o mejoraron en el momento posterior a la intervención. Se identificó la posibilidad de incluir diferencias individuales en la construcción y realización de prácticas que involucran intervenciones cognitivas, con el objetivo de una mayor efectividad de las técnicas utilizadas en el área de la salud.

Palabras clave: Evaluación psicológica; Envejecimiento; Salud.
Abstract
The objective was to analyze the effects of different cognitive interventions in two groups of elderly women, besides identifying the personality traits and their relations with cognition. Participants were 14 elderly women with a mean age of $64.79(\mathrm{SD}= \pm 3.57)$, with a high school or higher education. These elderly women belonged to a group of cognitive and physical 
interventions and were evaluated in two moments (preintervention and post-intervention), individually through an anamnesis, cognitive measures (Addenbrooke cognitive examination - revised version) and of personality (Factorial Battery of Personality). After the first evaluation, the participants were divided into groups of stimulation and cognitive training, and later, for the analysis of the results, it was decided to regroup the participants into two categories, being those that maintained/ improved or worsened in the results. The results indicated that low personality traits of instability, extraversion and social interactions were significant with participants who maintained or improved at the time of intervention. It was identified the possibility of including individual differences in the construction and conduct of practices involving cognitive interventions, aiming at a greater effectiveness of the techniques used in the health area.

Keywords: Psychological assessment; Aging; Health.

\section{Introdução}

O processo de envelhecimento humano se apresenta como um processo não apenas biológico, mas também histórico e cultural, a medida em que se observa, no decorrer da trajetória de vida, que com a idade se apresentam aspectos de declínio das capacidades físicas e cognitivas dos indivíduos, e que em meio a isso a historicidade do sujeito e a cultura na qual está inserido influem sobre o processo de envelhecimento e a própria concepção do que é ser idoso (Birman, 2015; Gehlen, Ventura, Stobäus, Bauer \& Vacaro, 2020).

Em meio ao processo de envelhecimento, tem se discutido a respeito de quais estratégias podem ser tomadas para se realizar a manutenção da performance cognitiva dos idosos e/ou recuperar determinadas funções que apresentam declínio cognitivo, especialmente em processos de construção de novas memórias e aprendizagens. Tais processos se baseiam em estratégias de intervenções cognitivas diversas que visam por meio de técnicas específicas para diferentes funções cognitivas, promover a neuroplasticidade em pessoas idosas (Brehmer, Kalpouzos, Wenger \& Lövdén, 2014; Chariglione, Janczura \& Belleville, 2018). Dentre estas estratégias de mediação destacamse duas modalidades: a estimulação cognitiva definida como o aperfeiçoamento de habilidades cognitivas e sociais dos indivíduos sem a utilização de técnicas específicas que visem determinadas funções cognitivas, tais como rodas de conversa e aulas sobre cuidados com a saúde; e o treino cognitivo, definido como a condução de técnicas e habilidades que tenham como foco promover a performance de funções cognitivas específicas, como por exemplo a memória (Mowszowski, Batchelor \& Naismith, 2010).

As práticas realizadas em treino cognitivo vêm demonstrando eficácia na sua proposta de apresentar melhorias na performance de determinadas funções cognitivas, especialmente, memória, habilidades visuoespaciais e fluência verbal entre grupos de idosos saudáveis ou que possuem déficits de memória comprovados (Maseda, Millán-Calenti, Lorenzo-López \& Núñez-Naveira, 2013; Butler et al., 2018; Boujut \& Belleville, 2019). Hyer et al., (2016) apresentam resultados semelhantes em grupos de idosos com déficits cognitivos leves, demonstrando que o grupo experimental obteve melhor performance nos questionários de atividades funcionais e de memória subjetiva, entretanto, o grupo controle também apresentou ganhos, este resultado foi descrito como parte da estimulação cognitiva ao qual foi exposto durante o processo de intervenção. A estratégia de treino cognitivo também apresentou ser significativa nas habilidades de grupos que tiveram treinamento em raciocínio e em velocidade, em comparação com o grupo controle. Outra característica observada foi que os grupos que receberam treinamento em memória, raciocínio e velocidade apresentaram um nível menor de dificuldades nas suas funções de atividades diárias no decorrer de 10 anos de estudo (Rebok et al., 2014).

Basak e O'Connell (2016) também apresentaram evidências da eficácia de intervenções cognitivas em estudo realizado com dois grupos, sendo um submetido a técnica 
de memória previsível e outro a técnica de memória imprevisível, observou-se que o treino de memória imprevisível se apresentou como uma opção mais eficaz como estratégia interventiva em idosos, possivelmente por requerer um maior controle cognitivo, embora essa compressão não seja definitiva. Entretanto, alguns estudos (Li et al., 2016; Sacramento \& Chariglione, 2019) ainda apresentam resultados que diferem de uma lógica do que poderia significar ser melhor, demonstrando que apenas combinar estratégias diferentes pode não ser a maneira mais eficaz de promover um aperfeiçoamento da performance cognitiva, lançando uma compreensão de que se deve analisar o contexto de aplicação e quais os papéis estas intervenções combinadas poderiam ter neste cenário.

Há ainda uma busca por compreender os efeitos comparativos entre intervenções cognitivas, e combinadas com outras atividades como a prática de exercícios físicos. Neste sentido, em uma revisão sistemática foi observado que a execução de intervenções cognitivas combinadas tem apresentado melhores resultados em comparação com o grupo controle e com somente a prática de exercícios físicos (Sacramento \& Chariglione, 2019). Entretanto, alguns estudos não encontraram significância entre intervenções combinadas e intervenções cognitivas (Zhu, Yin, Lang, He \& Li, 2016). Pesquisas em torno dos efeitos da prática de exercícios físicos e intervenções cognitivas em conjunto com estes, corroboram a ideia de que estas estratégias possuem um efeito benéfico no organismo dos indivíduos, demonstrando que o grupo que participou tanto do exercício físico quanto da intervenção cognitiva alcança melhores resultados, em comparação com o grupo que não recebeu estas estratégias de intervenção (Pothier et al., 2017; Nocera et al., 2020).

Uma outra vertente ainda muito recente tem buscado compreender como as diferenças individuais podem estar relacionadas com a cognição e as práticas de intervenções cognitivas. Neste sentido, a personalidade tem sido definida de diferentes maneiras ao longo da história, sofrendo modificações e aperfeiçoamentos a respeito da sua compreensão, portanto, existe a necessidade de uma definição a ser seguida para uma maior clareza e objetividade deste estudo. Desta forma, segundo Pervin e John (2003) "a personalidade representa aquelas características da pessoa que explicam padrões consistentes de sentimentos, pensamentos e comportamentos" (p. 23).

Um dos modelos mais utilizados para o estudo e compreensão da personalidade é o Modelo dos Cinco Grandes Fatores (Big Five/Five FactorModel). A partir de então, diversos estudos realizados comprovaram a eficácia deste modelo e contribuíram para o estabelecimento desta abordagem como válida e eficaz na compreensão da personalidade (Nunes, Hutz \& Nunes, 2016; Laros, Peres, Andrade, \& Passos, 2018; Tran, Wasserbauer \& Voracek, 2020).

De acordo com Hill, Kolanowski, Fick, Chinchilli e Jablonski (2014), a personalidade exerce influência sobre tarefas de recordação tardia e funções executivas para determinados idosos, com alto risco de declínio cognitivo, que participaram de um grupo de estimulação cognitiva. Observou-se que escores baixos de extroversão e abertura estiveram associados a uma maior atenção, enquanto baixa extroversão apresentou maior escore nas funções executivas, além de que, baixa extroversão também esteve associada a um maior escore nas tarefas de recordação tardia.

Determinados traços de personalidade, tais como o neuroticismo e a realização, demonstraram uma associação significativa com concentrações das proteínas betaamilóides, principalmente $\mathrm{o}$ traço de neuroticismo. Sujeitos com escores altos neste traço de personalidade e que possuíam uma pior memória subjetiva apresentavam maior concentração de beta-amilóides, podendo ser um indicativo de predição para a doença de Alzheimer (Snitz et al., 2015).

Contribuindo com estas perspectivas entre personalidade e cérebro, (Hock et al., 2014) observaram que escores altos do traço de 
neuroticismo apresentam relação com o declínio da performance cognitiva global, enquanto altos níveis do traço de realização apresentaram relação com uma maior eficácia nesta respectiva performance cognitiva e na tarefa de recordação imediata. Além destas características, escores altos no traço de socialização representaram uma melhora na performance da tarefa de recordação tardia. Ainda em relação a memória, alto neuroticismo tem se apresentado como associado a baixa modulação da neuroplasticidade na memória de trabalho, enquanto alta realização apresenta o papel inverso (Dima, Friston, Stephan \& Frangou, 2015).

Enquanto o neuroticismo costuma aparecer associado a um maior declínio cognitivo, alta realização e abertura se apresentam como relacionadas a uma melhor performance da memória e menor declínio cognitivo. Ainda neste estudo, observou-se que alta realização e baixa extroversão esteve associada a um menor declínio cognitivo em idosos (Luchetti, Terracciano, Stephan \& Sutin, 2016). Tais dados colaboram com estudos citados acima ao demonstrar que traços de neuroticismo tem uma tendência a serem encontrados em conjunto com o declínio cognitivo, enquanto baixa extroversão é comumente relacionada com uma melhor performance em diferentes funções cognitivas.

As características individuais se tornaram objeto de estudo daqueles que buscam entender como as diferenças pessoais podem predizer a forma como as pessoas interagem com o mundo. Neste sentido, apesar das diferenças culturais entre diferentes países como a França e os Estados Unidos, escores altos nos traços de personalidade de extroversão e abertura, predisseram comportamentos de alto engajamento em atividades físicas, sociais e cognitivas (Stephan, Boiché, Canada \& Terracciano, 2014). Assim, estudos relacionados a características de personalidade no campo da cognição, atividade física/esportes e saúde (Curtis, Windsor \& Soubelet, 2015; Griffin, Guillette \& Healy, 2015; Marissa et al., 2020), têm apresentado certas consistências em relação a determinados traços de personalidade, tais como o neuroticismo, realização e extroversão, e o que estes predizem sobre os sujeitos, no entanto, tais estudos ainda apresentam uma variabilidade muito grande no que diz respeito as demais categorias, socialização e abertura. Além disso, não apresentam definitivamente quais traços de personalidade podem ter maior influência em determinadas tarefas, uma vez que esta resposta se torna altamente dependente do contexto dos participantes e de experimentação, demonstrando haver ainda um amplo espaço para discussão e pesquisa a respeito do papel da personalidade na cognição e saúde.

Nesse levantamento observou-se uma limitação quanto aos estudos no âmbito nacional no que se refere a relação entre cognição e personalidade na população idosa. Nesse sentido, esse estudo sobre as relações entre personalidade e cognição em idosas no contexto brasileiro visa contribuir para um incremento na escassa literatura brasileira a respeito do assunto, bem como, lançar um olhar compreensivo sobre como a personalidade afeta a cognição neste cenário. Tais perspectivas podem auxiliar no desenvolvimento de técnicas específicas que auxiliem em intervenções cognitivas com a população idosa, favorecendo uma melhor qualidade das técnicas e resultados obtidos com e para este público.

Frente às informações apresentadas, o objetivo deste estudo foi compreender quais traços de personalidade estão relacionados com a cognição de idosas brasileiras, em um contexto de intervenção cognitiva, e como estes traços apresentaram ou não relação com os resultados dos testes de rastreio cognitivo em dois momentos distintos, sendo estes pré e pósintervenção.

\section{Método Participantes}

Participaram deste estudo 14 idosas do centro de convivência do idoso, secretaria de saúde e da comunidade, recrutadas pelo grupo de Pesquisa NeuroCog-idoso, com idades entre 
60 e 70 anos (Média $=64,79, \mathrm{DP}=3,57$ ), que possuem o ensino médio completo $(64,3 \%)$, ensino superior incompleto $(7,10 \%)$ e ensino superior completo $(28,60 \%)$. As participantes em sua maioria eram casadas $(42,9 \%)$ e de nível socioeconômico classificado em sua maioria nas classes B1 $(28,6 \%)$ e C1 $(28,6 \%)$, e todas participaram de um dos dois grupos de intervenção cognitiva. Tratou-se de uma amostra por conveniência.

Este estudo faz parte de uma pesquisa de âmbito maior intitulada "Avaliação de duas intervenções de memória em medidas fisiológicas, cognitivas e de humor em idosos do Distrito Federal.", desenvolvida pelo grupo NeuroCog-Idoso. Tendo sido aprovado pelo Comitê de Ética em Pesquisa sob o número de parecer: 2.071.414, CAAE: 67653517.4.0000.0029.

Este recorte aqui apresentado teve como critérios de inclusão ter idade igual ou superior a 60 anos; participar da primeira avaliação de anamnese e das medidas cognitivas (para a verificação do desempenho em uma linha de base); participar das intervenções cognitivas (para o acompanhamento de diferentes intervenções cognitivas); ter ensino médio completo ou superior (para o acompanhamento da medida de personalidade); não possuir alguma psicopatologia autorrelatada; participar da aplicação da bateria fatorial de personalidade e participar da segunda avaliação de medidas cognitivas (para a verificação do comportamento dessas variáveis em função do tempo).

\section{Instrumentos}

$\mathrm{O}$ primeiro instrumento a ser apresentado foi o Termo de Consentimento Livre e Esclarecido (TCLE), de forma com que pudessem aceitar a participação voluntária no estudo. Após a apresentação e mediante a aceitação do TCLE, foram utilizados os seguintes materiais com os participantes, a serem descritos a seguir:

- Roteiro de Anamnese. Utilizou-se deste protocolo para a construção do perfil socioeconômico, de saúde, escolarização, estado civil, sexo e demais informações relativas à amostra.

\section{- Exame Cognitivo de Addenbrooke -} Versão revisada. Este instrumento de rastreio cognitivo, adaptado para a população brasileira, apresenta elevada sensibilidade na avaliação de diferentes funções cognitivas, dentre elas: Atenção e orientação, memória, fluência verbal, linguagem e habilidades visuoespaciais. O Exame Cognitivo de Addenbrooke - Versão revisada (ACE-R) possui cinco domínios, cada um com uma pontuação específica, sendo: Atenção e Orientação (pontuação total de 18 pontos e nota de corte de 17 pontos); Memória (pontuação total de 26 pontos e nota de corte de 15 pontos); Fluência (pontuação total de 14 pontos e nota de corte de 8 pontos); Linguagem (pontuação total de 26 pontos e nota de corte de 22 pontos) e Visuoespacial (pontuação total de 16 pontos e nota de corte de 13 pontos), conforme Carvalho e Caramelli, 2007.

- Bateria Fatorial de Personalidade. Trata-se de teste psicológico de vasta evidência de validade e confiabilidade, capaz de promover um alcance amplo de público-alvo no contexto brasileiro. Sendo composto por 126 itens que avaliam diferentes fatores de personalidade, cujos cincos principais fatores são: Neuroticismo, abertura, realização, socialização e extroversão, além de outros fatores (Nunes, Hutz \& Nunes, 2016).

\section{Intervenções}

As intervenções cognitivas consistiram em duas modalidades distintas de intervenção, a seguir apresenta-se a descrição das práticas de cada um dos grupos:

A técnica de treino cognitivo (MEMO) consiste na aprendizagem de estratégias de memória e de atenção, conforme orientações de Belleville et al., (2018). Esta técnica caracteriza-se por intervenções baseadas no treino cognitivo, com atividades estruturadas, que permitem o aprimoramento diferentes subsistemas nos domínios de atenção e memória, por meio de diferentes técnicas 
como, por exemplo: o método de Loci, o método de associação nome-rosto e o método PQRST (Preview, Question, Read, Sumarize, Test).

A técnica de estimulação cognitiva (Stimullus) utilizou de intervenções psicossociais e educativas, sem estar associada a uma técnica específica que visasse determinada função cognitiva. A estimulação cognitiva é relatada como capaz de auxiliar na proteção do declínio cognitivo (Chariglione \& Janczura, 2013). As atividades incluíram sequência visual, memorização de palavras, memorização de frases, categorização de palavras, memorização de imagens e memorização de estórias com recuperação imediata e tardia.

\section{Procedimentos \\ $1^{\text {a }}$ Etapa - Inscrição dos participantes}

Este encontro consistiu em apresentar o projeto e suas perspectivas de resultados para a população idosa do Centro de Convivência do Idoso da Universidade Católica de Brasília, dos postos da Secretaria de Saúde e demais associados da instituição. As inscrições foram feitas presencialmente através de uma lista de presença cedida pela equipe de estudo e pesquisa NeuroCog-Idoso. Essa etapa do estudo aconteceu em junho/2017.

\section{$2^{\text {a }}$ Etapa - $1^{\text {a }}$ Avaliação Cognitiva}

As avaliações ocorreram em agosto/2017, sendo apenas um encontro com cada participante, com uma duração média de uma hora. Foi apresentado o termo de consentimento livre e esclarecido aos participantes antes do início desta primeira etapa de avaliações. Durante o período de avaliação foram aplicados diversos testes, mas sendo importante para esta análise a Anamnese e o ACE-R. Foi considerado um período de avaliação, para cada participante, de até 30 dias antes do início das intervenções cognitivas com o objetivo de minimizar as variáveis intervenientes.

\section{$3^{\text {a }}$ Etapa - Intervenções Cognitivas}

Os participantes deste estudo foram inseridos em dois grupos de intervenção, sendo sete participantes no grupo de estimulação cognitiva (Stimullus) e sete participantes no treino cognitivo (MEMO). Estas intervenções tiveram duração de seis semanas, durante este período as atividades ocorreram uma vez por semana com duração de 1 h30 cada sessão. Essa etapa do estudo aconteceu entre setembro e outubro/2017.

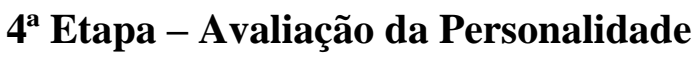

Após o fim das intervenções cognitivas, foram realizadas as avaliações da personalidade dos participantes através do uso da Bateria Fatorial de Personalidade. Estas sessões tiveram duração média de uma hora, sendo realizada individualmente e em apenas uma sessão. Essa avaliação aconteceu em novembro/2017, 30 dias após as intervenções cognitivas, com o objetivo de minimizar as variáveis intervenientes.

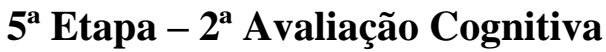

Após finalizadas as intervenções cognitivas e tendo sido aplicada a Bateria Fatorial de Personalidade, os participantes foram reavaliados através do ACE-R. Essa avaliação ocorreu em apenas um encontro com cada participante, com duração média de 1 hora. Essa avaliação aconteceu em fevereiro/2018, com pelo menos seis meses após a primeira avaliação cognitiva. Esse cuidado metodológico visou minimizar os efeitos de aprendizagem, na medida em que os testes utilizados não possuíam diferentes versões para aplicação.

\section{Análise de dados}

Os dados foram analisados utilizando o programa SPSS-IBM (versão 23), devidamente registrado. Primeiramente foram calculadas as estatísticas descritivas da amostra, em seguida foi verificada a normalidade dos dados entre grupos de estimulação cognitiva e treino cognitivo através do Shapiro-Wilk, que se apresentaram como normais. A partir disso, foi feito o Teste $t$ para amostras emparelhadas do 
ACE-R em dois momentos distintos, em seguida foi calculado o delta dos participantes e feito o teste t para amostras independentes a partir do delta das categorias entre grupos.

Em seguida, optou-se por não distinguir mais entre grupos de estimulação e treino cognitivo, mas sim, por reagrupar os participantes e abarcar toda a amostra entre pessoas que mantiveram ou melhoraram e aqueles que pioraram nos resultados, para isso foi feito um novo teste para verificar a normalidade (Shapiro-Wilk) dos dados entre traços de personalidade e estes respectivos grupos. Após constatado que não havia normalidade nos dados dos grupos dos que mantiveram/melhoraram ou pioraram, optou-se por utilizar o teste de Mann-Whitney para identificar em quais traços de personalidade estes dois grupos se diferem significativamente. $\mathrm{O}$ nível de significância estipulado foi de $\mathrm{p} \leq 0,05$.

\section{Resultados}

Participaram deste estudo 14 idosas com idade média de 64,79 anos ( $\mathrm{DP}=3,57)$, todos do sexo feminino, com ensino médio completo $(64,30 \%)$, ensino superior incompleto $(7,10 \%)$ e ensino superior completo $(28,60 \%)$. O estado civil das participantes é definido como casadas $(42,90 \%)$, divorciadas $(35,70 \%)$ e viúvas $(21,40 \%)$. O nível socioeconômico dos participantes está entre as classes A2 e C2, sendo que $21,40 \%$ estão na classe A2, 28,60\% (B1), 14,30\% (B2), 28,60\% (C1) e 7,10\% (C2), segundo a escala de classificação socioeconômica da Associação Brasileira de Empresas de Pesquisa (ABEP), conforme a Tabela1.

Tabela 1.

Caracterização sociodemográfica da Amostra $(N=14)$

\begin{tabular}{cccc}
\hline \multirow{2}{*}{ Características } & & Média & Desvio Padrão \\
\cline { 3 - 4 } & Idade & 64,79 & 3,57 \\
\cline { 3 - 4 } Escolaridade & Ensino médio & 9 & Porcentagem \\
& Ensino superior incompleto & 1 & 64,30 \\
& Ensino superior & 4 & 7,10 \\
& Casada & 6 & 28,60 \\
\hline \multirow{2}{*}{ Estado civil } & Divorciada & 5 & 42,90 \\
& Viúva & 3 & 35,70 \\
& A2 & 3 & 21,40 \\
\hline \multirow{2}{*}{ ABEP } & B1 & 4 & 28,60 \\
& B2 & 2 & 14,30 \\
& C1 & 4 & 28,60 \\
& C2 & 1 & 7,10 \\
\hline
\end{tabular}

Nota. Variável idade representada por média e desvio padrão, as variáveis escolaridade, estado civil e ABEP por suas frequências e porcentagem.

Para além da caracterização da amostra, foram realizadas análises de comparação entre os diferentes momentos (pré e pós-intervenção) e o comportamento da variável cognitiva (ACE-R), conforme a Tabela 2.

Observou-se na Tabela 2 que os resultados pré e pós teste do ACE-R (sem a distinção entre os grupos) apresentaram melhora nas médias das seguintes categorias: Memória; fluência verbal; linguagem; habilidades visuoespaciais e total no teste. No entanto, o teste t para amostras emparelhadas demonstrou que essa melhora no grupo como um todo não é significativa $(\mathrm{p} \geq 0,005)$.

Uma vez observado que houve uma melhora na maioria dos escores do grupo como um todo e que apesar disso essa melhora não foi estatisticamente significativa, optou-se por analisar o delta das categorias da amostra para identificar quais grupos mantiveram o mesmo nível de pontuação ou melhoraram em relação ao pré-teste, bem como, se houve significância nos resultados entre grupos, conforme a Tabela 3 . 
Tabela 2.

Resultados do Teste t para Amostras Emparelhadas do ACE-R em Dois Momentos Distintos da Amostra $(N=14)$

\begin{tabular}{cccccccc}
\hline & \multicolumn{2}{c}{ Pré } & & \multicolumn{2}{c}{ Pós } & & Sig. Pré e Pós \\
\cline { 2 - 3 } Categorias & Média & Desvio padrão & & Média & Desvio padrão & & p-valor \\
\hline Atenção e orientação & 17,28 & 1,13 & & 16,78 & 2,32 & & 0,47 \\
Memória & 20,78 & 3,23 & & 21,57 & 3,45 & & 0,33 \\
Fluência verbal & 10,85 & 1,74 & & 11,35 & 1,69 & & 0,26 \\
Linguagem & 23,35 & 4,41 & & 23,64 & 3,41 & & 0,50 \\
Visuoespaciais & 14,21 & 2,11 & & 14,28 & 2,33 & & 0,89 \\
Total & 86,50 & 8,19 & & 87,64 & 10,73 & & 0,38 \\
\hline
\end{tabular}

Tabela 3.

Resultados do Teste t para Amostras Independentes a partir do Delta das Categorias entre Grupos $(N=14)$

\begin{tabular}{ccccccc}
\hline \multirow{2}{*}{ Delta das categorias } & Grupos & $\mathrm{N}$ & Média & Desvio padrão & $\begin{array}{c}p \text {-valor } \\
\text { Atenção e orientação }\end{array}$ & $\begin{array}{c}\text { Melhoraram/ } \\
\text { mantiveram }\end{array}$ \\
& Estimulação cognitiva & 7 & 0,71 & 1,49 & 0,07 & $100 \%$ \\
\multirow{2}{*}{ Memória } & Treino cognitivo & 7 & $-1,71$ & 2,92 & 0,08 & $42,86 \%$ \\
& Trtimulação cognitiva & 7 & 0,57 & 3,50 & 0,79 & $71,43 \%$ \\
& Treino cognitivo & 7 & 1,00 & 2,44 & 0,79 & $71,43 \%$ \\
\hline \multirow{2}{*}{ Fluência verbal } & Estimulação cognitiva & 7 & 0,57 & 2,22 & 0,87 & $71,43 \%$ \\
& Treino cognitivo & 7 & 0,42 & 0,78 & 0,87 & $85,71 \%$ \\
\hline \multirow{2}{*}{ Linguagem } & Estimulação cognitiva & 7 & 0,28 & 0,48 & 1,00 & $100 \%$ \\
& Treino cognitivo & 7 & 0,28 & 2,21 & 1,00 & $71,43 \%$ \\
\hline \multirow{2}{*}{ Visuoespaciais } & Estimulação cognitiva & 7 & $-0,85$ & 1,34 & 0,09 & $42,86 \%$ \\
& Treino cognitivo & 7 & 1,00 & 2,30 & 0,09 & $85,71 \%$ \\
\hline \multirow{2}{*}{ Total } & Estimulação cognitiva & 7 & 1,28 & 5,99 & 0,91 & $71,43 \%$ \\
& Treino cognitivo & 7 & 1,00 & 3,65 & 0,91 & $71,43 \%$ \\
\hline
\end{tabular}

Conforme apresentado na Tabela 3, a análise do delta de cada uma das categorias através do Teste t para amostras independentes, demonstrou que não houve nenhuma diferença significativa entre os resultados do treino cognitivo e da estimulação cognitiva. No entanto, observou-se que ao verificar a categoria do delta total do ACE-R, 71,43\% dos participantes dos grupos de estimulação cognitiva e treino cognitivo se mantiveram no nível ou melhoraram. Neste sentido, apesar de não apresentarem diferenças se pertencentes ao grupo estimulação cognitiva ou treino cognitivo, se faz importante destacar que as duas estratégias promoveram uma tendência a estabilidade ou melhoraram os resultados da amostra entre grupos.

Em seguida optou-se por analisar a amostra total $(n=14)$, entre participantes que mantiveram ou melhoraram e os que pioraram nos resultados (Tabela 4). Portanto, foram divididos nos grupos dos que mantiveram/melhoraram e os que pioraram. Essa escolha se deu após ter sido observado que não houve diferenças significativas entre o tipo de intervenção e os resultados apresentados de acordo com a Tabela 3 . 
Tabela 4.

Resultados do Teste de Mann-Whitney Entre Grupos que Melhoraram/Mantiveram ou Pioraram $(N=14)$

\begin{tabular}{cccccc}
\hline $\begin{array}{c}\text { Traços de } \\
\text { personalidade }\end{array}$ & Grupos & $\mathrm{N}$ & Média & Desvio padrão & $p$-valor \\
\hline \multirow{2}{*}{ Instabilidade } & Melhoraram/Mantiveram & 10 & 2,73 & 0,83 & 0,04 \\
& Pioraram & 4 & 3,79 & 0,79 & 0,04 \\
\hline \multirow{2}{*}{ Extroversão } & Melhoraram/Mantiveram & 10 & 4,40 & 0,45 & 0,01 \\
& Pioraram & 4 & 5,19 & 0,60 & \multirow{2}{*}{$0,0,69$} \\
\hline Interações & Melhoraram/Mantiveram & 10 & 4,95 & 0,60 & 0,21 \\
sociais & Pioraram & 4 & 6,21 & 0 \\
\hline
\end{tabular}

Conforme os resultados apresentados na Tabela 4, os traços de personalidade de instabilidade, extroversão e interações sociais apresentaram índices bastante significativos. Neste sentido, o grupo dos que mantiveram/melhoraram tem escores baixos dos traços de personalidade de instabilidade (Média $=2,73, \mathrm{DP}=0,83$ ), extroversão (Média $=4,40, \mathrm{DP}=0,45)$ e interações sociais (Média $=4,95, \mathrm{DP}=0,69)$, que integra $\mathrm{o}$ fator 'extroversão', em comparação com o grupo dos que pioraram.

\section{Discussão}

Diante do processo de envelhecimento observa-se uma importante possibilidade de surgimento de déficits cognitivos, entretanto, estas transformações podem ser afetadas por uma série de fatores, tais como o nível de escolaridade, a saúde física e psicológica, o ambiente, e atualmente, cada vez mais estudos têm destacado as características de personalidade. Neste sentido, todos estes fatores podem afetar a qualidade de vida das pessoas idosas de diferentes formas, seja de modo a contribuir no declínio cognitivo ou como fatores protetivos para um envelhecimento cognitivo mais saudável. Nessa perspectiva, o envelhecimento cognitivo não é apenas um "desenvolvimento ao contrário" e oferece oportunidades de reflexões mais abrangentes das funções cognitivas e as suas modificações ao longo do tempo (Craik \& Bialystok, 2006).

Assim, verifica-se uma concepção de envelhecimento em transformação nas últimas décadas (Birman, 2015; Gehlen et al., 2020), oferecendo novos olhares para esta etapa da vida, não apenas marcada pelas perdas, mas também pelos ganhos nesse processo que também pode ser de desenvolvimento.

A partir desta perspectiva de desenvolvimento e com o objetivo de compreender a associação de traços de personalidade em idosos brasileiras observouse, nessa amostra, um escore médio maior na maioria das medidas na avaliação pósintervenção, especificamente nas categorias memória, fluência verbal, linguagem, habilidades visuoespaciais e no escore total do teste. Embora as médias obtidas nestas categorias tenham aumentado no pós-teste em relação ao pré-teste, tais diferenças não foram significativas $(\mathrm{p} \geq 0,005)$. No entanto, essas médias corroboram estudos anteriores que indicam uma importante tendência ao incremento cognitivo em determinados testes com presença ou ausência de grupo controle nessas comparações ( $\mathrm{Li}$ et al., 2016; Sacramento \& Chariglione, 2019).

Ainda se observou, nessa amostra, que o tipo de intervenção adotada, seja ela de estimulação cognitiva (Stimullus) ou de treino cognitivo (MEMO), não apresentaram diferenças significativas no que se refere a ganhos cognitivos, resultados corroborados por Chariglione, Janczura e Belleville (2018) e Sacramento e Chariglione (2019). Entretanto, estes resultados não devem ser compreendidos como uma ausência de ganho nos grupos, mas 
que ambas as estratégias de intervenção cognitiva ofereceram o mesmo nível de ganho aos participantes, também conforme apresentados nos estudos supracitados.

Em relação aos grupos terem obtido ganhos similares, tais resultados encontram respaldo na literatura, uma vez que estudos comparativos entre diferentes tipos de intervenções apresentam que em um primeiro momento, grupos que participaram das práticas interventivas obtiveram ganhos, em determinadas categorias avaliadas, logo após as intervenções, no entanto, participantes que realizaram sessões de treino cognitivo apresentam escores mais altos em testes cognitivos por um período maior de tempo do que aqueles que apenas realizaram sessões de estimulação cognitiva (Rahe et al., 2015; Pothier et al., 2017).

No que se refere a personalidade entende-se que esta pode exercer um papel modulador nas características cognitivas dos indivíduos que participam de intervenções cognitivas, tais influências sugerem uma importante associação com melhores ou piores resultados em diferentes tarefas de memória, atenção, função executiva e mesmo em características de ação física/motora (Hill et al., 2014; LeMonda, Mahoney, Verghese, \& Holtzer, 2015; Snitz et al., 2015; Nocera et al., 2020).

A partir da realização do teste de MannWhitney (Tabela 4), foi observado que três características apresentavam diferenças significativas entre os grupos que mantiveram/melhoraram e os que pioraram. Tais características são: instabilidade, extroversão e interações sociais. A instabilidade é uma subcategoria do traço de neuroticismo, e está associada a irritabilidade, nervosismo e variações de humor. Pessoas com traços de extroversão apresentam tendências a serem mais comunicativos, expansivos, ativos e buscarem comportamentos de liderança, além destes, as interações sociais são uma subcategoria do traço de extroversão, e está associada a participação de atividades em grupo, interações com outras pessoas e facilidade para se envolver com outras pessoas (Nunes, Hutz \& Nunes, 2016).

Quando observadas as médias na Tabela 4, verificou-se que o grupo dos que mantiveram/melhoraram tem escores mais baixos em instabilidade, extroversão e interações sociais, em comparação com o grupo dos que pioraram. A extroversão é citada na literatura como um traço em que baixos níveis estão associados a melhor performance cognitiva em diferentes tarefas como memória, atenção e funções executivas. Além disto, a extroversão também aparece relacionada com a resiliência de atletas, onde atletas mais extrovertidos tendem a participar de esportes em grupo e os autores relatam possuírem menos controle antes de agir (LeMonda et al., 2015; Luchetti et al., 2016; Ceviker, Ozlu, Deryahanoglu, Demirdoken \& Turkay, 2020). Não foram encontrados estudos que relacionassem os traços de instabilidade e interações sociais com a cognição em idosos.

Portanto, tendo como base na literatura estudada, compreende-se que a menor presença destes respectivos três traços de personalidade, podem ter influenciado, sobre os participantes do grupo mantiveram/melhoraram, uma maior capacidade de concentração, atenção e planejamento da sua participação nas intervenções cognitivas, bem como, a presença de um menor nível de instabilidade pode estar relacionado a uma maior capacidade de lidar com frustrações decorrentes dos desafios e tarefas das intervenções. Como uma subcategoria do traço de neuroticismo, a influência de um maior nível do traço de instabilidade na cognição dos participantes do grupo piorou, pode estar de acordo com a literatura associado com o declínio cognitivo e baixa performance cognitiva deste grupo de participantes (Hock et al., 2014; LeMonda et al., 2015; Snitz et al., 2015; Margioti et al., 2020). O traço de interações sociais foi o aspecto que apresentou maior significância de acordo com a Tabela 4, e os participantes do grupo que mantiveram/melhoraram tiveram uma menor média neste traço, que pode ter se traduzido durante as intervenções em participantes que buscavam interagir menos um 
com os outros, aumentando assim sua capacidade de se concentrar nas atividades e técnicas elaboradas durante as intervenções cognitivas.

\section{Conclusão}

Compreende-se que os objetivos deste estudo foram parcialmente alcançados, mas sendo importante destacá-lo como um importante estudo exploratório das relações entre personalidade, cognição e intervenções cognitivas com idosas no âmbito brasileiro. Observa-se que os resultados encontrados podem auxiliar na prática de futuras intervenções realizadas por pesquisadores e profissionais da saúde, além de contribuir com a literatura nacional a respeito das associações entre características de personalidade e intervenções cognitivas em idosos. Essas reflexões buscam minimizar as perdas amostrais e favorecer técnicas que se adaptem melhor às diferenças individuais.

No decorrer deste estudo, observou-se a possibilidade de se produzir sugestões futuras, tais como a replicação destes procedimentos com uma amostra maior. Uma nova metodologia que tenha como objetivo compreender os diferentes efeitos que as intervenções cognitivas podem ter sobre os participantes, quais destas apresentam melhores resultados a longo prazo e como a personalidade pode estar relacionada com estes efeitos de longo prazo. Outra sugestão a ser citada, se refere a construção e normatização de testes psicológicos que avaliem a personalidade de populações idosas, uma vez observada que existem poucas opções que possam ser utilizadas com esta população.

Entende-se que a busca por uma melhor compreensão de como a personalidade e a cognição podem se relacionar, especialmente em estudos com a população idosa, exige ainda um longo caminho de estudos e avanços científicos. Porém, o aprimoramento dessa perspectiva teórico-prática pode ser primordial para a composição dos grupos segundo as suas características de personalidade e o tipo de intervenção, promovendo assim, espaços mais coerentes com as habilidades a serem treinadas e o tipo de técnica a ser aplicada.

\section{Referências}

Basak, C., \& O'Connell, M. A. (2016). To

Switch or Not to Switch: Role of Cognitive Control in Working Memory Training in Older Adults. Front Psychol, 7, 230. doi: 10.3389/fpsyg.2016.00230

Belleville, S., Hudon, C., Bier, N., Brodeur, C., Gilbert, B., Grenier, S., ... Gauthier, S. (2018). MEMO+: Efficacy, Durability and Effect of Cognitive Training and Psychosocial Intervention in Individuals with Mild Cognitive Impairment. J Am Geriatr Soc, 66(4), 655-663. doi: 10.1111/igs.15192

Birman, J. (2015). Terceira idade, subjetivação e biopolítica. História, Ciências, Saúde, 22(4), 1267-1282. doi: 10.1590/S0104$\underline{59702015000400007}$

Boujut, A., \& Belleville, S. (2019). Où en eston avec les programmes d'interventions cognitives pour les personnes âgées?.
Revue de neuropsychologie, 11(1), 60-69.

Recuperado de

https://www.cairn.info/revue-revue-deneuropsychologie-2019-1-page-60.htm

Brehmer, Y., Kalpouzos, G., Wenger, E., \& Lövdén, M. (2014). Plasticity of brain and cognition in older adults. Psychol Res, 78(6), 790-802. doi: 10.1007/s00426-014$0587-\mathrm{Z}$

Butler, M., McCreedy, E., Nelson, V. A., Desai, P., Ratner, E., Fink, Kane, R. L. (2018). Does cognitive training prevent cognitive decline? A systematic review. Annals of Internal Medicine, 168, 63-68. doi: 10.7326/M17-1531

Carvalho, C. A., \& Caramelli, P. (2007). Brazilian adaptation of the Addenbrooke's Cognitive Examination-Revised. Dement. Neuropsychol, 1(2), 212-216. doi: $\underline{10.1590 / \mathrm{s} 1980-57642008 \mathrm{dn} 10200015}$ 
Ceviker, A., Ozlu, K., Deryahanoglu, G., Demirdoken, C., \& Turkay, H. (2020). The examination of the personality traits and optimal performance mood of the university athletes. Pedagogy of Physical Culture and Sports, 24(1), 4-11. doi: 10.15561/18189172.2020.0101

Chariglione, I. P. F., \& Janczura, G. M. (2013) Contribuições de um treino cognitivo para a memória de idosos institucionalizados.

Psico-USF, 18(1), 13-22. doi: 10.1590/S1413-82712013000100003

Chariglione, I. P. F. S., Janczura, G. A., \& Belleville, S. (2018). Cognitive interventions to improve memory in healthy older adults: the use of Canadian (MEMO) and Brazilian (Stimullus) approaches. Estudos de Psicologia (Natal), 23(1), 2-13. doi: 10.22491/16784669.20180002

Craik, F. I. and Bialystok, E. (2006). Cognition through the lifespan: mechanisms of change. Trends in Cognitive Sciences, 10, 131-138. doi: 10.1016/j.tics.2006.01.007

Curtis, R. G., Windsor, T. D., \& Soubelet, A. (2015). The relationship between Big-5 personality traits and cognitive ability in older adults - a review. Neuropsychol Dev Cogn B Aging Neuropsychol Cogn, 22(1), 42-71. doi: 10.1080/13825585.2014.888392

Dima, D., Friston, K. J., Stephan, K. E., \& Frangou, S. (2015). Neuroticism and conscientiousness respectively constrain and facilitate short-term plasticity within the working memory neural network. Hum Brain Mapp, 36(10), 4158-4163. doi: 10.1002/hbm.22906

Gehlen, M., Ventura, J., Stobäus, C., Bauer, M., \& Vacaro, J. (2020). Historical dialectical materialism in the learning of the elderly person in the promotion of health care. Research, Society and Development, 9(2), e156922214. doi: 10.33448/rsd-v9i2.2214

Griffin, A. S., Guillette, L. M., \& Healy, S. D. (2015). Cognition and personality: an analysis of an emerging field. Trends Ecol
Evol, 30(4), 207-214. doi:

10.1016/j.tree.2015.01.012

Hill, N. L., Kolanowski, A. M., Fick, D., Chinchilli, V. M., \& Jablonski, R. A. (2014). Personality as a moderator of cognitive stimulation in older adults at high risk for cognitive decline. Res Gerontol Nurs, 7(4), 159-170. doi: 10.3928/19404921-20140311-01

Hock, R. S., Lee, H. B., Bienvenu, O. J., Nestadt, G., Samuels, J. F., Parisi, J. M., Jr, P. T. C., \& Spira, A. P. (2014). Personality and cognitive decline in the Baltimore Epidemiologic Catchment Area follow-up study. Am J Geriatr Psychiatry, 22(9), 917925. doi:10.1016/j.jagp.2012.12.217

Hyer, L., Scott, C., Atkinson, M. M., Mullen, C. M., Lee, A., Johnson, A., \& Mckenzie, L. C. (2016). Cognitive Training Program to Improve Working Memory in Older Adults with MCI. Clin Gerontol, 39(5), 410-427. doi: 10.1080/07317115.2015.1120257

Laros, J. A., Peres, Al. J. S., Andrade, J. M., \& Passos, M. F. D. (2018). Validity evidence of two short scales measuring the Big Five personality factors. Psicologia: Reflexão $e$ Crítica, 31(32). doi: 10.1186/s41155-0180111-2

LeMonda, B. C., Mahoney, J. R., Verghese, J., \& Holtzer, R. (2015). The Association between High Neuroticism-Low Extraversion and Dual-Task Performance during Walking While Talking in Nondemented Older Adults. J Int Neuropsychol Soc, 21(7), 519-530. doi: $10.1017 / \mathrm{S} 1355617715000570$

Li, B., Zhu, X., Hou, J., Chen, T., Wang, P., \& Li, J. (2016). Combined Cognitive Training vs. Memory Strategy Training in Healthy Older Adults. Front Psychol, 7, 834. doi: 10.3389/fpsyg.2016.00834

Luchetti, M., Terracciano, A., Stephan, Y., \& Sutin, A. R. (2016). Personality and Cognitive Decline in Older Adults: Data From a Longitudinal Sample and MetaAnalysis. J Gerontol B Psychol Sci Soc Sci, 71(4), 591-601. doi: 10.1093/geronb/gbu184 
Margioti, E., Kosmidis, M. H., Yannakoulia, M., Dardiotis, E., Hadjigeorgiou, G., Sakka, P., ... Scarmeas, N. (2020). Exploring the association between subjective cognitive decline and frailty: the Hellenic Longitudinal Investigation of Aging and Diet Study (HELIAD). Aging \& Mental Health, 24(1), 137-147. doi: 10.1080/13607863.2018.1525604

Marissa A. G., Courtland S. H., Kharine R. J., Violeta J. R., Talia L. R. \& Lloyd S. M. (2020). A Multi-method Investigation of the Personality Correlates of Functional Ability in Older Adults. Clinical Gerontologist, 43(4), 363-364, doi: 10.1080/07317115.2019.1709239

Maseda, A., Millán-Calenti, J. C., LorenzoLópez, L., \& Núñez-Naveira, L. (2013). Efficacy of a computerized cognitive training application for older adults with and without memory impairments. Aging Clin Exp Res, 25(4), 411-419. doi: 10.1007/s40520-013-0070-5

Mowszowski, L., Batchelor, J., \& Naismith, S. L. (2010). Early intervention for cognitive decline: can cognitive training be used as a selective prevention technique? Int Psychogeriatr, 22(4), 537-548. doi: 10.1017/S1041610209991748

Nocera, J. R., Mammino, K., Kommula, Y., Wharton, W., Crosson, B., \& McGregor, K. M. (2020). Effects of Combined Aerobic Exercise and Cognitive Training on Verbal Fluency in Older Adults. Gerontology and Geriatric Medicine,6, 1-11-. doi: 10.1177/2333721419896884

Nunes, C. H. S., Hutz, C. S., \& Nunes, M. (Eds.). (2016). Bateria Fatorial de Personalidade (BFP): Manual Técnico. São Paulo: Casa do Psicólogo.

Pervin, L. A., \& John, O. P. (Eds.). (2003). Personalidade: Teoria e Pesquisa. Brasil: ArtMed.

Pothier, K., Gagnon, C., Fraser, S. A., Lussier, M., Desjardins-Crépeau, L., Berryman, N., ... Bherer, L. (2017). A comparison of the impact of physical exercise, cognitive training and combined intervention on spontaneous walking speed in older adults.
Aging Clin Exp Res., 30, 921-925. doi: 10.1007/s40520-017-0878-5

Rahe, J., Petrelli, A., Kaesberg, S., Fink, G. R., Kessler, J., \& Kalbe, E. (2015). Effects of cognitive training with additional physical activity compared to pure cognitive training in healthy older adults. Clin Interv Aging, 10, 297-310. doi: 10.2147/CIA.S74071

Rebok, G. W., Ball, K., Guey, L. T., Jones, R. N., Kim, H. Y., King, J. W., ... Willis, S. L. (2014). Ten-year effects of the advanced cognitive training for independent and vital elderly cognitive training trial on cognition and everyday functioning in older adults. $J$ Am Geriatr Soc, 62(1), 16-24. doi: 10.1111/jgs.12607

Sacramento, A. M., \& Chariglione, I. P. F. S. (2019). Intervenções físicas e cognitivas combinadas para melhora cognitiva no envelhecimento: Uma revisão sistemática. Revista Brasileira de Psicologia do Esporte, 9(1), 47-63. doi: 10.31501/rbpe.v9i1.9965

Snitz, B. E., Weissfeld, L. A., Cohen, A. D., Lopez, O. L., Nebes, R. D., Aizenstein, H. J., ... Klunk, W. E. (2015). Subjective Cognitive Complaints, Personality and Brain Amyloid-beta in Cognitively Normal Older Adults. Am J Geriatr Psychiatry, 23(9), 985-993. doi: 10.1016/j.jagp.2015.01.008

Stephan, Y., Boiché, J., Canada, B., \& Terracciano, A. (2014). Association of personality with physical, social, and mental activities across the lifespan: Findings from US and French samples. $\mathrm{Br} J$ Psychol, 105(4), 564-580. doi: 10.1111/bjop.12056

Tran, U. S., Wasserbauer, J., \& Voracek, M. (2020). Testing the incremental validity of dispositional mindfulness over and above the Big Five in accounting for mental health: A facet-level structural-equation modeling and predictor communality and dominance approach. Personality and Individual Differences, 156, 109769. doi: 10.1016/j.paid.2019.109769

Zhu, X., Yin, S., Lang, M., He, R., \& Li, J. (2016). The more the better? A meta- 
analysis on effects of combined cognitive and physical intervention on cognition in healthy older adults. Ageing Res Rev, 31, 67-79. doi: $10.1016 / \mathrm{j}$. arr.2016.07.003

\section{Dados sobre os autores:}

- Caio Diogo Santana de Sousa: Psicólogo pela Universidade Católica de Brasília, Brasília - DF, Brasil. Membro do Grupo de Pesquisa CNPq NeuroCog-Idoso.

- Isabelle Patriciá Freitas Soares Chariglione: Psicóloga, Doutora em Cognição e Neurociências pela Universidade de Brasília. Professora de Psicologia no Departamento de Psicologia Escolar e do Desenvolvimento pela Universidade de Brasília, Brasília - DF, Brasil. Coordenadora do Grupo de Pesquisa CNPq NeuroCog-Idoso.

\section{Agradecimentos:}

- Essa presente pesquisa foi realizada graças ao apoio e aprovação no: 0193.001227/2016 da Fundação de Apoio à Pesquisa do Distrito Federal - FAPDF.

Declaração de Direito Autoral

A submissão de originais para este periódico implica na transferência, pelos autores, dos direitos de publicação impressa e digital. Os direitos autorais para os artigos publicados são do autor, com direitos do periódico sobre a primeira publicação. Os autores somente poderão utilizar os mesmos resultados em outras publicações indicando claramente este periódico como o meio da publicação original. Em virtude de sermos um periódico de acesso aberto, permite-se o uso gratuito dos artigos em aplicações educacionais e científicas desde que citada a fonte conforme a licença CC-BY da Creative Commons. 